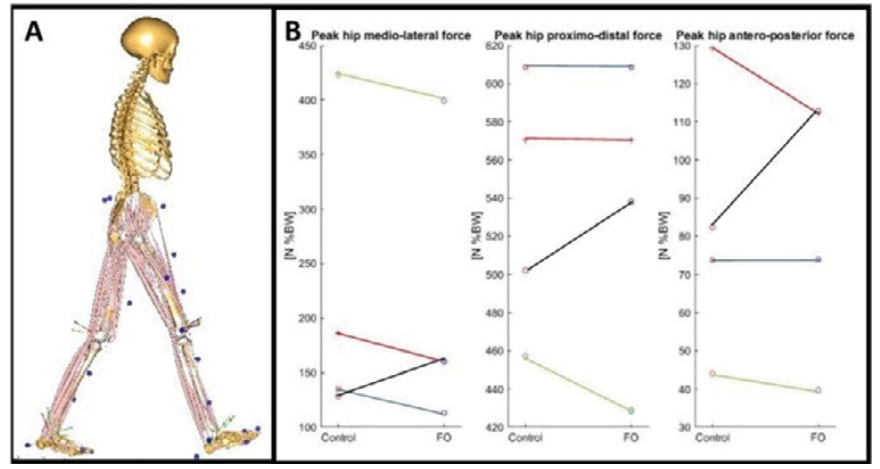

acquired from MRI [3]. Accurate joint centers and axes were calculated with analytical surface fits to the segmented MRI bones for the hip, knee and ankle. Results: Peak hip force for medio-lateral (ML) proximo-distal (PD) and antereoposterior $(\mathrm{AP})$ is presented in Figure $1 \mathrm{~B}$ for the $\mathrm{C}$ and $\mathrm{FO}$ with values for each subject.

Conclusions: The results of this study indicate that FO can change the load distribution in the hip joint. A reduction or similar values for ML, DP and AP force was found for all but one participant. These changes may potentially contribute to the reduction in pain. Further studies are needed to investigate if there is a relationship between changed loading and pain for RA patients. This knowledge can potential be used for design of better FO and clinical guidelines for use of FO. References:

[1] Hennessy K. et al, Arthritis Care Res, 64: 311-320, 2012.

[2] Lewinson R. et al., Ann Biomed Eng, 44: 3173-3185, 2016.

[3] Marra M, et al., J Biomech Eng. 137: BIO-14-1490, 2015.

Acknowledgements: The study is financially supported by The Danish Rheumatism Association [R142-A4113].

Disclosure of Interest: None declared

DOI: 10.1136/annrheumdis-2017-eular.2169

\section{THU0716 LONGITUDINAL OUTCOME OF AEROBIC FITNESS IN ADOLESCENTS AND YOUNG ADULTS WITH JIA}

P.V. Pelt ${ }^{1,2}$, T. Takken ${ }^{3}$, M.V. Brussel ${ }^{4}$, R. Dolhain ${ }^{1}$, J. Hazes ${ }^{1}$, J. Bijlsma ${ }^{5}$ N. Wulffraat ${ }^{6}$, A. Kruize ${ }^{5}$. ${ }^{1}$ Department of Rheumatology, Erasmus MC, University Medical Center Rotterdam, Rotterdam; ${ }^{2}$ Department of Pediatric Immunology, Wilhelmina Children's Hospital, University Medical Center Utrecht; ${ }^{3}$ Child Development and Exercise Center, Wilhelimina Children's Hospital, University Medical Center Utrecht; ${ }^{4}$ Child Development and Exercise Center, Wilhelmina Children's Hospital, University Medical Center Utrecht; ${ }^{5}$ Department of Rheumatology and Immunology, University Medical Center Utrecht; ${ }^{6}$ Department of Pediatric Immunology, Wilhelmina Children's Hospital, University Medical Center Utrecht, Utrecht, Netherlands

Background: Aerobic fitness may serve as an important health-related outcome measure in JIA. A reduced aerobic fitness is associated with cardiovascular morbidity, mortality and osteoporosis in adult patients with chronic diseases. However, in adolescents and young adults, long-term outcome data of aerobic fitness are unknown. Reduced aerobic fitness was described in cross-sectional studies in children and adolescents with $\mathrm{JIA}$, and was more impaired in active disease Objectives: Our objectives are to describe course of the aerobic fitness in a longitudinal cohort of adolescents and young adult JIA-patients who are intensively treated including the possibility of biologics and to identify the association of clinical variables with aerobic fitness.

Methods: In a longitudinal cohort, all consecutive JIA patients aged 10-24 years were included after informed consent. Annual examinations were obtained from demographic and disease-related items. At baseline and end of the study, aerobic fitness (VO2peak) was assessed using a graded cardiopulmonary exercise test (CPET) to volitional exhaustion performed on an electronically braked cycle ergometer. Absolute and relative VO2-peak values were measured and related to healthy controls (Z-scores), using one-sample T-tests. Non-parametric tests were used to evaluate results

Results: Paired Z-scores were available from 27 patients. $44 \%$ were male, median age at baseline was 13,0yrs (IQR 4,3), disease duration 7,6yrs $(6,7)$, JADAS27 $4,0(5,9)$, DAS28 2,2 (1,2). 76\% of the patients were in DAS28-remission. $11 \%$ had systemic JIA, 7\% persistent oligoarticular and $82 \%$ had a polyarticular course. Baseline and end Z-scores were reduced compared to healthy controls (ZAbs_base -0,68, IQR2,3 p=0,01; Zrel_base -1,33, IQR 2,0, p $<0,01$; Zabs_end $-0,23$, IQR 1,7, $p=0,06$; Zrel_end $-0,87, \mathrm{IQR} 2,2, \mathrm{p}=0,01$ ) and did not change significantly over time (change Zabs change $0,45, \mathrm{p}=0,34$; Zrel change 0,46 , $p=0,31)$. At baseline, MTX-use $(p=0,04)$ and a higher DAS28 $(p=0,015)$ and ESR $(p=0,013)$ are associated with a worse outcome of aerobic fitness. The greatest improvement of aerobic fitness over time was seen in patients with a higher ESR $(p<0,01)$ and thrombocytes $(p<0,01)$ at baseline. Multivariate analysis showed that a higher DAS28 and male gender were the most important variables for worse aerobic fitness at baseline, a higher ESR at baseline was the most important predictor for improving aerobic fitness over time

Conclusions: Aerobic fitness is significantly reduced in adolescents and young adults with JIA and does not improve over time, despite intensive treatment. Be aware of a reduced quality of life due to a persistent reduced aerobic fitness during disease course of JIA, despite low disease activity

\section{References:}

[1] Aerobic capacity and disease activity in children, adolescents and young adults with juvenile idiopathic arthritis (JIA). Ph.A. van Pelt MD, T. Takken PhD, M.van Brussel PhD, M. de Witte. A.A. Kruize*, MD, N.M.Wulffraat* MD, PhD. Pediatr Rheumatol Online J. 2012 Aug 27;10(1):27. doi: 10.1186/1546-0096-10-27. Disclosure of Interest: None declared

DOI: 10.1136/annrheumdis-2017-eular.4756

\section{THU0717 MIRROR VISUAL FEEDBACK THERAPY IMPROVES CLINICAL OUTCOMES AND THE ACTIVITY OF DAILY LIVING TO PATIENTS WITH HAND COMPLEX REGIONAL PAIN SYNDROME}

$\underline{\text { S. Patru }}^{1}$, I.R. Marcu ${ }^{2}$, D. Matei ${ }^{1}$, A.M. Bumbea ${ }^{1}$, A.C. Bighea ${ }^{1} .{ }^{1}$ Physical Medicine and Rehabilitation; ${ }^{2}$ University of Medicine and Pharmacy of Craiova, Craiova, Romania

Background: Wrist osteoporotic fractures may be as disabling as vertebral or hip fractures and the functional decline attributed to them is similar to that seen for arthritis and diabetes mellitus. Sometimes these fractures will lead to a painful, debilitating condition with sensory and motor disturbances, changes in vascular tone, temperature and edema- complex regional pain syndrome (CRPS). We found some clinical trials and case reports which conclude that mirror visual feedback therapy (MVFT) improves clinical outcomes and the activity of daily living to patients with hand neurological disorders, including CRPS.

Objectives: In this controlled randomized clinical study we tried to investigate the effects of (MVFT) in CRPS type I following osteoporosis wrist fracture.

Methods: We included 21 subjects with osteoporotic wrist fracture and early CRPS (duration of 3-4 weeks), with a single hand affected by allodynia, stiffness and vasomotor disturbances, from Physical Medicine and Rehabilitation outpatient clinic. They are randomly assigned into two groups: MVFT group ( $n=11$, simply place a mirror between their two hands and train the patient by asking them to move both hands while watching the reflection of the non-affected hand in the mirror, 10 minutes for each session, four times a day) and Control group $(n=10$, moved both hands separated by an opaque partition between the arms). All subjects also received conventional therapy. On presentation and after 4 weeks of rehabilitation programme we assessed the wrist flexion and extension with a goniometer and the Patient-Rated Wrist Evaluation (PRWE) a 15-items questionnaire designed to measure wrist pain and disability in activities of daily living.

Results: Subjects in the mirror therapy group showed significant improvement in range of motion: extension increased with $50.4 \%$ vs. $41.7 \%$ and for flexion MVFT achieved $33.2 \%$ and Control group $16.8 \%(P<.001)$. The rehabilitation programme also increased hand function with better results to 4 weeks PRWE for MVFT group (40.4 vs. $51.8, P=0.003$ )

Conclusions: MVF is a simple, inexpensive, without adverse events treatment option that significantly reduces pain and stiffness and improves hand mobility in early CRPS after osteoporosis wrist fracture.

\section{References:}

[1] Vladimir Tichelaar YI, et al. Mirror box therapy added to cognitive behavioural therapy in three chronic complex regional pain syndrome type I patients: a pilot study. Int J Rehabil Res 2007; 30: 181-8.

[2] Bache SJ, Ankcorn L, Hiller L, Gaffrey A. Two different approaches to physiotherapy management of patients with distal radius fractures. Physiotherapy. 2000;86:383.

Disclosure of Interest: None declared

DOI: 10.1136/annrheumdis-2017-eular.2339

\section{THU0718 DIFFERENCES IN THE COURSE OF ITALIAN- AND GERMAN-SPEAKING PATIENTS' OUTCOME AFTER INTERDISCIPLINARY PAIN PROGRAM}

T. Benz ${ }^{1,2}$, S. Lehmann ${ }^{2}$, R. Brioschi ${ }^{2}$, A. Elfering ${ }^{1}$, A. Aeschlimann ${ }^{2}$ F. Angst ${ }^{2}$. ${ }^{1}$ Institute of Psychology, University of Bern, Bern; ${ }^{2}$ Research Department, RehaClinic, Bad Zurzach, Switzerland

Background: Available evidence shows that the experience and perception of pain varies among different populations. Further, inequalities are reported in pain treatment across various types of pain and in different settings (1). In particular, it is unknown how much immigrants in Western European countries profit from pain management programs.

Objectives: The aim of this study was to detect differences in the course of Italianand German-speaking patient's state of health and quality of life after a 4-week standardized interdisciplinary pain management program in German or in Italian. Methods: The prospective cohort study with 61 Italian-speaking and 63 Germanspeaking patients with fibromyalgia or chronic back pain measured health-related quality of life, pain, anxiety and depression comparing at baseline, after 4 weeks of pain program and at 1 year follow-up. Differences between the two groups 
were tested on significance by generalized estimation equations (GEE) (2). This method modeled changes of health by multivariate logistic regression adjusting for sex, education, number of comorbidities and the baseline score over both follow-ups for each scale.

Results: Italian-speaking patients $(n=61)$ showed higher proportions of males, lower educated and less burdened by comorbidities than German-speaking patients $(n=63)$. At baseline, physical and psychosocial health, depression and anxiety of the Italian-speaking patients were worse than German-speaking patients, with the exception of less pain in the Italian-speaking patients on the SF-36. Changes of health showed more improvement in German- than in Italian-speaking patients on all scales and at both follow-ups. In GEE, the highest differences were observed in SF-36 physical functioning ( $p=0.035)$, HADS anxiety $(p=0.038)$ and HADS depression $(p=0.023)$. On SF-36 bodily pain, the difference was not significant $(\mathrm{p}=0.166)$.

Conclusions: This study detected that short- and midterm outcome of Italianspeaking patients was worse than that of German-speaking patients, even after adjustment for baseline differences. The reasons for this study's results remain unclear, but may have consequences for future management of Italian-speaking patients in interdisciplinary pain management programs. Considering language as a proxy for acculturation, this supports the hypothesis that patients with lower level of acculturation may have special needs in therapeutic management. A cultural sensitive approach in a multidisciplinary pain program might enhance the positive outcome in the short- and mid-term (3).

[1] Green CR, Anderson KO, Baker TA, Campbell LC, Decker S, Fillingim RB, Kalauokalani DA, Lasch KE, Myers C, Tait RC, Todd KH, Vallerand AH. The unequal burden of pain: confronting racial and ethnic disparities in pain. Pain Med 2003;4:277-94.

[2] Twisk JW. Generalized estimating equations. Applied longitudinal data analysis for epidemiology. 2nd ed. Cambridge: Cambridge University Press; 2013: p 57-68.

[3] Benz T, Lehmann S, Brioschi R, Elfering A, Aeschlimann A, Angst F. Comparison of short- and mid-term outcome of Italian- and German-speaking patients after an interdisciplinary pain management program. Disabil Rehabil 2017; in preparation.

Disclosure of Interest: None declared

DOI: 10.1136/annrheumdis-2017-eular.5888

\section{THU0719 LONG-TERM COURSE OF HEALTH AND WORKING CAPACITY AFTER A REHABILITATION PROGRAM FOR WHIPLASH INJURY}

T. Benz ${ }^{1}$, P. Haiduk ${ }^{1}$, S. Lehmann ${ }^{1}$, F. Gysi-Klaus ${ }^{1}$, B.A. Michel ${ }^{2}$

A. Aeschlimann ${ }^{1}$, F. Angst ${ }^{1} .{ }^{1}$ Research Department, RehaClinic, Bad Zurzach;

${ }^{2}$ Clinic of Rheumatology, University Hospital of Zurich, Zurich, Switzerland

Background: Persistent pain and disability of whiplash injury associated disorders (WAD) cause high burden for the individual and costs for healthcare.
Objectives: The aim of this study was to determine state and change of health and working-capacity five years after a standardized inpatient pain management program of four weeks.

Methods: This prospective cohort study quantified health and quality of life by the generic Short Form 36 (SF-36, 100=best), the neck-specific Northern American Spine Society (NASS) form, and the Coping Strategies Questionnaire (CSQ). SF-36 data were compared to age-, sex-, and comorbidity-specific German population norms (1). Changes of health were determined using effect sizes (ES) at the 6 month and the 60 month follow-up. Changes of health were determined using effect sizes (ES) (2).

Results: The 59 participants had mean age of 40.3 years ( $s d=12.3$ ), $83 \%$ were women, and $37 \%$ had one or more comorbidites. At 5 years, health was worse on all SF-36 scales when compared to the norms $(p<0.001)$, varying from mean 41.5 , norm 82.3 on role physical to mean 65.7 , norm 71.0 on mental health (all $\mathrm{p}<0.001)$

Table 1

Effect sizes (ES) Entry to 60 months 6 months to 60 months

SF-36 Physical functioning

\begin{tabular}{ll}
0.99 & 0.16 \\
2.22 & 0.83 \\
1.61 & 0.78 \\
0.89 & 0.32 \\
0.71 & 0.47 \\
0.61 & 0.30 \\
1.12 & 0.56 \\
0.78 & 0.26 \\
1.03 & 0.62 \\
\hline
\end{tabular}

SF-36 Role physical

SF-36 Bodily pain

SF-36 Vitality

SF-36 Social functioning

SF-36 Mental health

NASS Pain

NASS Function

CSQ Catastrophizing

Median working capacity improved from 0 at entry to 21 at 6 months and to 30 hours/week at 5 years.

Conclusions: Moderate to large long-term effects were observed. Substantial improvements still occurred between 6 and 60 months after start of the pain program, especially in pain, catastrophizing, and physical role performance. Improvements observed after the inpatient pain program can be maintained and expanded in the long-term at home (3).

References:

[1] Kurth BM, Ellert U. The SF-36 questionnaire and its usefulness in population studies: Results of the German Health Interview and Examination Survey 1998. Soz Praeventivmed 2002:47:266-277.

[2] Kazis ES, Anderson JJ, Meenan RF. Effect sizes for interpreting changes in health status. Med Care 1989;27(3 Suppl):S178-89.

[3] Haiduk P, Benz T, Lehmann S, Gysi-Klaus F, Aeschlimann A, Michel BA, Angst F. Interdisciplinary rehabilitation after whiplash injury: An observational prospective five year outcome study. Medicine 2017; in press.

Disclosure of Interest: None declared

DOI: 10.1136/annrheumdis-2017-eular.6178 